\title{
Predictors of Surgical Candidacy in 414 Epilepsy Patients Admitted to the EMU
}

\author{
Alireza Mansouri, Aria Fallah, George M. Ibrahim, Taufik A. Valiante
}

\begin{abstract}
Objectives: Admission to an Epilepsy Monitoring Unit (EMU) is essential for pre-surgical evaluation of patients with medically-refractory epilepsy; however, prolonged referral times and resource limitations are significant access barriers. Therefore, identification of pre-EMU variables that predict potential surgical candidates can assist in the triage of patient admissions to the EMU. Methods: In this hypothesis-generating study, a retrospective analysis of patients admitted for pre-surgical evaluation to the Toronto Western Hospital EMU (2004-2011) was performed. Univariate and multivariate logistic regression was used to identify variables that could independently predict subsequent surgical candidacy following EMU evaluation. Results: Four hundred and fourteen patients were admitted to the EMU. Overall, 259 patients (62.5\%) were identified as potential surgical candidates. One hundred and seven patients $(25.8 \%)$ required invasive electroencephalogram (iEEG) implantations; of 75 patients consenting to iEEG analysis 39 underwent a subsequent resective procedure. Male patients and those with a lesion on MRI were 1.9 times more likely to be surgical candidates (95\% CI 1.18-2.98 and 0.94-3.80, respectively), while patients with non-localizable seizures were seven times less likely (95\% CI 0.02-1.25). Conclusion: In this retrospective, hypothesis-generating study male gender, presence of a lesion on MRI and localizable seizures on routine outpatient EEG analysis independently predicted subsequent resective epilepsy surgical candidacy in EMU patients. Upon validation by other studies, these variables may be considered by clinicians referring patients to the EMU in order to improve wait times and optimize patient care.
\end{abstract}

RÉSUMÉ: Facteurs de prédiction caractérisant les candidats à la chirurgie chez 414 patients atteints d'Épilepsie admis à l'usé. Objectif : Il est essentiel d'admettre les patients présentant une épilepsie réfractaire au traitement médical à une unité de surveillance de l'Épilepsie (usé) pour une évaluation avant la chirurgie. Cependant, le temps d'attente et les ressources limitées constituent des obstacles importants. L'identification de variables qui sont des facteurs de prédiction caractérisant les candidats à la chirurgie avant leur admission à l'usé pourrait aider au triage préadmission. Méthode : Dans cette étude dont le but était de générer des hypothèses nous avons analysé rétrospectivement les dossiers des patients admis pour une évaluation préchirurgicale à l'usé du Toronto Western Hospital de 2004 à 2011. Nous avons utilisé l'analyse de régression univariée et multivariée pour identifier les variables qui pouvaient prédire de façon indépendante que le patient serait un candidat à la chirurgie après l'évaluation à l'usé. Résultats : Quatre cent quatorze patients ont été admis à l'usé. Parmi ces patients, 259 patients $(62,5 \%)$ ont été identifiés comme étant des candidats potentiels à la chirurgie. cent sept patients $(25,8 \%)$ ont eu besoin d'un ÉEG effractif. Soixante-quinze patients ont consenti à cet examen et 39 d'entre eux ont subi une résection par la suite. Les hommes et ceux qui avaient une lésion à l'IRM étaient 1,9 fois plus susceptibles d'être des candidats à la chirurgie (IC à $95 \%$ : 1,18 à 2,98 et 0,94 à 3,80 respectivement), alors que les patients qui avaient des crises non localisables étaient sept fois moins susceptibles de l'être (IC à $95 \%$ : 0,02 à 1,25). Conclusion : Dans cette étude rétrospective destinée à générer des hypothèses, le sexe masculin, la présence d'une lésion à l'irm et des crises localisables à l'ÉEG de routine effectué en externe chez des patients admis à l'usé étaient des facteurs de prédiction indépendants d'une candidature à une résection chirurgicale. Après validation par d'autres études, les cliniciens qui réfèrent des patients à l'usé pourraient tenir compte de ces variables afin de diminuer le temps d'attente et d'optimiser les soins aux patients.

Can J Neurol Sci. 2013; 40: 372-377

Approximately one third of patients with epilepsy fail to respond to anti-epileptic drugs ${ }^{1}$, a subset of whom benefit from surgical resection ${ }^{2}$. The surgical assessment typically includes at minimum scalp electroencephalography (EEG) and magnetic resonance imaging (MRI). In addition, admission to the Epilepsy Monitoring Unit (EMU), where ictal and inter-ictal activity detected via continuous EEG are used in conjunction with video monitoring to characterize and localize epileptogenic cortex, is the cornerstone of all comprehensive epilepsy programs ${ }^{3}$. Following EMU evaluation, the decision may be made to proceed with resective surgery, attempt to localize the seizures further via implantation of invasive EEG (iEEG) electrodes or other means, or the patient may be considered a non-surgical candidate altogether ${ }^{4}$. In each of these scenarios, an admission to the EMU is a fundamental component in the pre-surgical evaluation.
Access to epilepsy surgery remains a considerable challenge in contemporary healthcare systems ${ }^{5}$. The average delay between a diagnosis of medically intractable epilepsy and referral for surgical consideration and surgery can be as long as 15-22 years ${ }^{6,7}$. Once referred for pre-surgical evaluation, an

From the Division of Neurosurgery (AM, AF, GMI, TAV), Institute of Medical Sciences (GIM, TAV), University of Toronto; University Health Network (TAV), Division of Fundamental Neurobiology, Toronto Western Research Institute (TAV), Toronto Western Hospital; Krembil Neuroscience Center (TAV), Toronto; Department of Clinical Epidemiology and Biostatistics (AF), McMaster University, Hamilton, Ontario, Canada.

Received September 5, 2012. Final Revisions Submitted November 23, 2012. Correspondence to: Alireza Mansouri, Toronto Western Hospital, 4W-436, 399 Bathurst Street, Toronto, Ontario, M5T 2S8, Canada.

Email: alireza.mansouri@utoronto.ca. 
Table 1: Patient demographics and summary of univariate analysis for patients admitted to the EMU

\begin{tabular}{|c|c|c|c|}
\hline Variable & Median (IQR) & Odds ratio $(95 \% \mathrm{CI})$ & P value \\
\hline Age at first seizure (years) & $5(0) \pm 9.8$ & $2.2(0.14-36.0)$ & 0.58 \\
\hline Age at EMU analysis (years) & $37(38) \pm 11.3$ & $1.01(1.00-1.03)$ & 0.11 \\
\hline Age at first surgery (years) & $40(42) \pm 12.8$ & $\mathrm{~N} / \mathrm{A}$ & N/A \\
\hline Variable & Frequency (Percentage) & Odds ratio $(95 \% \mathrm{CI})$ & P value \\
\hline Gender (Male) & $207(50 \%)$ & $1.4(0.9-2.1)$ & 0.11 \\
\hline History of febrile seizures & $24(5.8 \%)$ & $1.9(0.9-4.6)$ & 0.11 \\
\hline Failure to localize seizures on pre-EMU EEG & $7(1.7 \%)$ & $0.2(0.0-1.9)$ & 0.13 \\
\hline Lesion on MRI & $341(82.4 \%)$ & $1.7(0.9-3.3)$ & 0.07 \\
\hline Invasive EEG electrode implantation & $73(17.6 \%)$ & $1.6(1.0-2.7)$ & 0.07 \\
\hline
\end{tabular}

additional waiting period is required for evaluation of patients in EMUs. At our institution, this delay is approximately 17 months. In general, upon completion of the EMU assessment, approximately half of the patients are offered resective surgery ${ }^{8}$. Given the limitations in resources and demand for EMU assessments, information that can be used to expedite the process is of great value.

Here, we sought to identify variables prior to EMU admission that may be associated with candidacy for resective epilepsy surgery following analysis within the EMU. As a secondary objective, we also analyzed predictors of surgical candidacy in patients who underwent invasive EEG monitoring. Overall, the aim of the current study is to identify variables that can be further analyzed and validated in future studies with the ultimate goal of assisting clinical decision making with regards to the triage of EMU admissions.

\section{MethodS}

\section{Patient population}

A retrospective chart review of patients referred for presurgical evaluation at the Toronto Western Hospital (TWH) EMU from 2004 to 2011 was performed. The TWH is an adult tertiary referral center (one of two centers in Ontario, Canada), with a five bed EMU. The epilepsy team consists of one epilepsy surgeon and five epileptologists. The database was compiled during weekly multi-disciplinary epilepsy patient management conferences, which are held following each patient's EMU admission. The variables collected for each patient in this database were identified by the surgeon (TAV) to be potentially associated with patients undergoing subsequent resective surgery. Patient demographics and variables collected in the database are presented in Table 1. Certain patients required iEEG monitoring for better characterization of seizures. At our center, the criteria for iEEG monitoring are an inability to localize seizures and/or the epileptogenic regions are felt to be close to eloquent cortex.

\section{Outcomes measured and statistical analysis}

The primary outcome of interest was whether or not a patient admitted to the EMU was considered a candidate for resective surgery. Independent variables included in the study were limited to a sample of convenience comprised of: Age at first seizure and at EMU admission, patient gender, history of febrile seizures, and the presence of lesion on MRI. The ability to electrographically localize seizures on pre-EMU outpatient EEG was also analyzed.

Post EMU admission variables that were extracted included whether or not seizures were electrographically detectable on EEG during the EMU stay and if so whether they were localizable, lateralizable, multifocal, bilateral, or involving eloquent cortex. Furthermore, the number of patients who were adequately controlled with medication (contrary to their status at time of referral), those with psychogenic non-epileptic seizures (PNES), and those lost to follow-up was also recorded.

Variables with a frequency of fewer than five occurrences were excluded from the final statistical model. Continuous variables are expressed as means, standard deviations and ranges while nominal and ordinal variables are expressed as frequencies and percentages. Univariate analysis was performed to identify potential predictors of patients undergoing resective epilepsy surgery (Table 1). Covariates with a p value of less than 0.2 on univariate analysis were selected for multivariate regression (Table 2). Multivartiate logistic regression (stepwise forward

Table 2: Summary of multivariate analysis to identify predictors of surgical candidacy following admission to the EMU

\begin{tabular}{l|c|c}
\hline & $\begin{array}{c}\text { Odds ratio } \\
\mathbf{( 9 5 \%} \mathbf{C I})\end{array}$ & P value \\
\hline Lesion on MRI & $1.89(0.94-3.80)$ & 0.07 \\
\hline Gender (Male) & $1.88(1.18-2.98)$ & 0.01 \\
\hline Failure to localize seizures & $0.14(0.02-1.25)$ & 0.08 \\
\hline
\end{tabular}




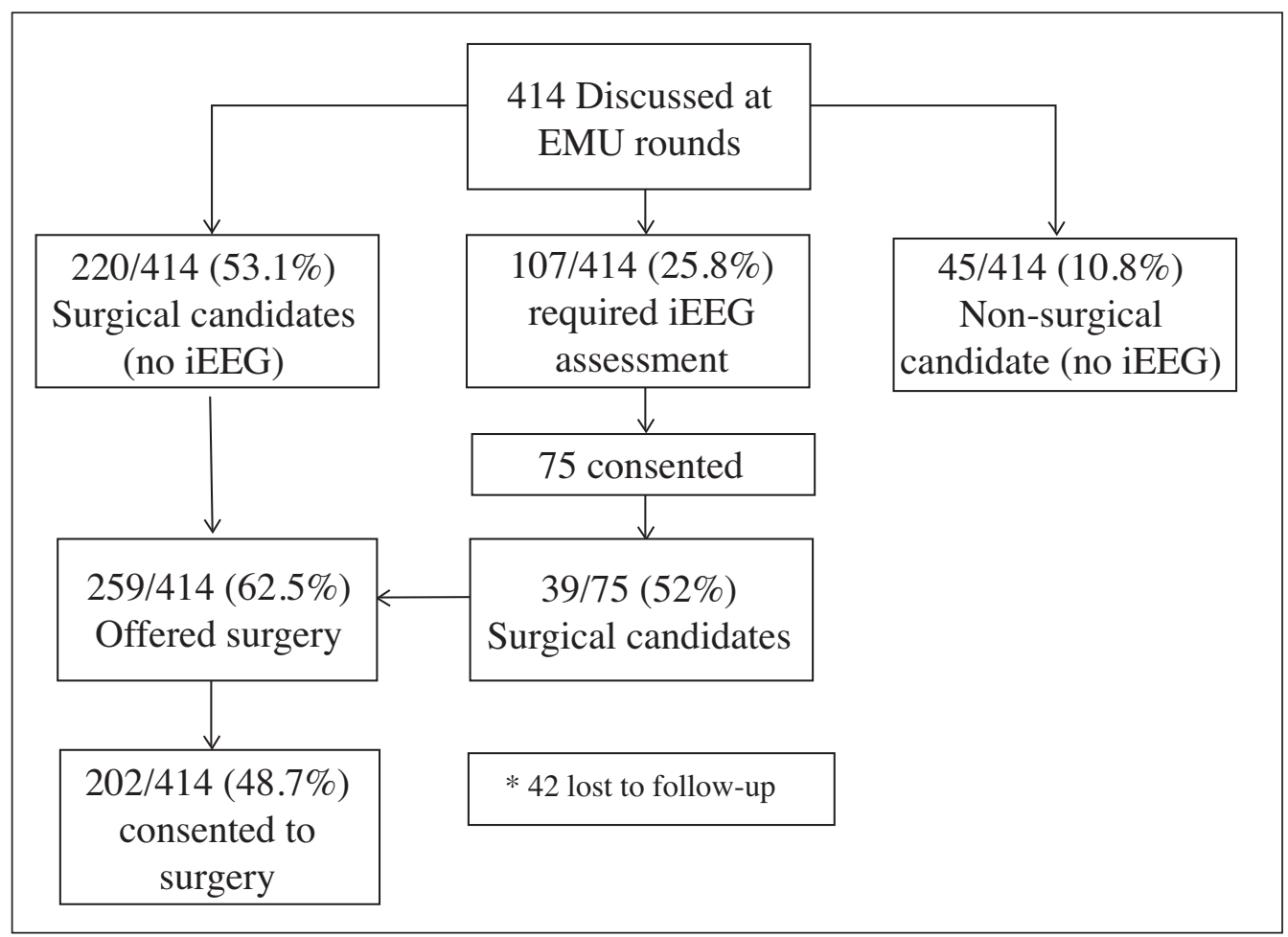

Figure 1: Flowchart of patients assessed in the EMU at TWH between the years 2004-2011.

conditional method) was performed to identify independent predictors of patients undergoing resective epilepsy surgery. As this was a hypothesis-generating exercise, we considered covariates with a $\mathrm{p}$ value of less than 0.10 in our multivariate regression to be a potential independent predictor of resective epilepsy surgery.

\section{RESUlts}

\section{EMU evaluation}

Our database contained information collected consecutively on a total of 417 patients; 414 patients had complete records and were therefore included in the analyses (Figure 1). In total, 259 patients $(62.5 \%)$ were offered resective surgery upon completion of EMU assessment; most of these patients were identified without the need for invasive monitoring. Meanwhile, 107 patients $(25.8 \%)$ required iEEG implantation to further localize the seizures. Of this cohort, 14 refused invasive monitoring altogether and 18 remained undecided at the time of this study. Of the 75 patients who consented to iEEG implantation, 39 $(52 \%)$ underwent subsequent resective epilepsy surgery. One patient who was deemed a surgical candidate died unexpectedly, likely secondary to Sudden Unexpected Death in Epilepsy (SUDEP) prior to the resective procedure. Forty two patients (10\% overall) were lost to follow-up.

Among all candidates, 71 (including those who declined invasive monitoring) declined surgical intervention following an informed consent discussion. Thus, patient preference was the mitigating factor for the exclusion of $47 \%$ of the 152 patients not undergoing resective surgery. In the remaining 81 patients
(53\%), the decision not to offer surgery was made by the epilepsy team (Figure 2A).

\section{Factors associated with subsequent resective surgery following EMU admission}

The primary reason for the exclusion of patients from surgical consideration was an inability to identify the seizure focus (48 patients; 60\%). Within this cohort, 17 patients (35\%) had seizures that were non-localizable/lateralizable, while multifocal or bilateral seizure foci were identified in 14 (30\%) and 17 $(35 \%)$ of patients, respectively (Figure 2B). Among the remaining 33 patients, 11 (14\%) had epilepsy involving eloquent cortex, $10(13 \%)$ had achieved adequate seizure control with medication, 6 (7\%) were diagnosed with PNES, and electrographic seizures were not captured in $6(7 \%)$.

Univariate analysis of all patients admitted to the EMU identified six variables (male gender, a history of febrile seizures, the presence of a lesion on MRI, a failure to localize seizures on pre-admission EEG, the need for invasive electrode implantation, and age at EMU admission) as potential predictors of surgical candidacy (Table 1). We also sought to identify possible predictors of surgical candidacy in patients undergoing iEEG monitoring. In this group, univariate analysis revealed a trend that those with a history of febrile seizures were 3.8 times more likely to go on to a resective procedure (95\% CI 0.7-19.2, $\mathrm{p}=0.11$ ). However, given the wide confidence interval and the concept that febrile seizures are intimately associated with Mesial Temporal Sclerosis (MTS), we did not consider this to be clinically significant. 


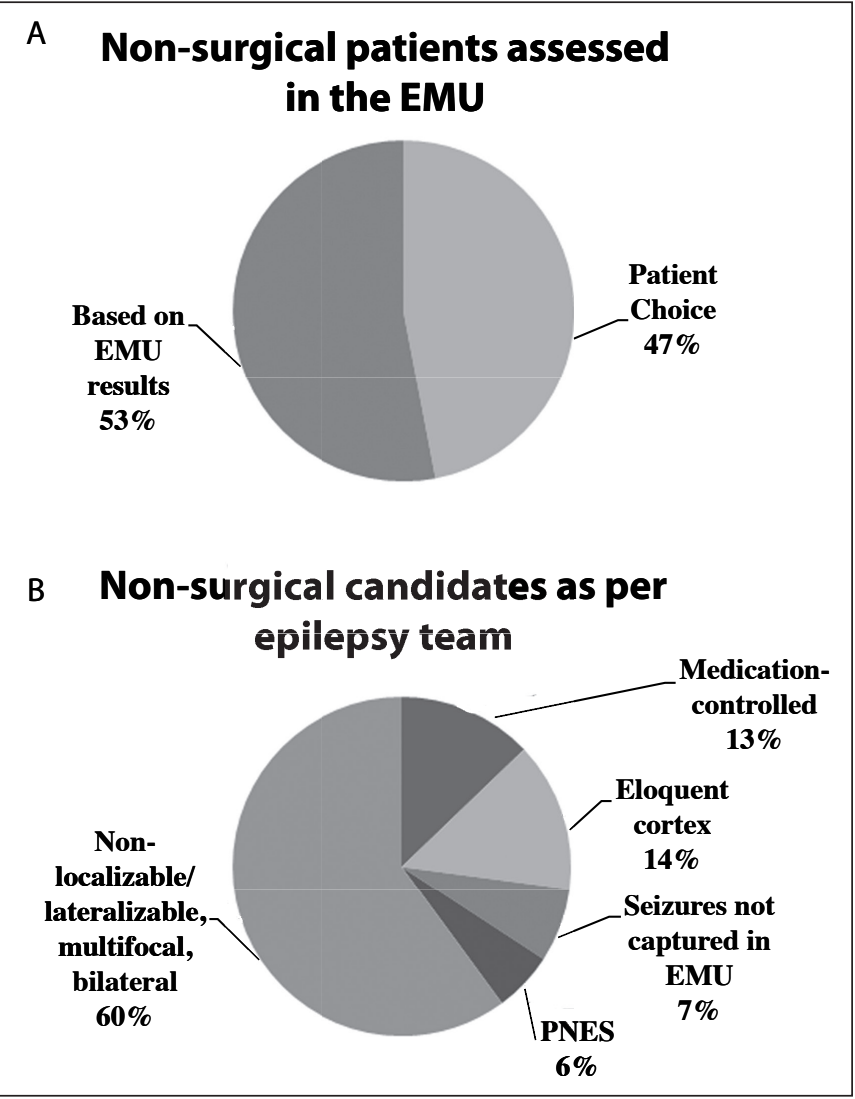

Figure 2: A) Analysis of the overall reasons for patients not undergoing surgical resection for medically-intractable epilepsy. Patient choice includes patients who refused surgery for iEEG implantation. B) Analysis of factors affecting decision by the epilepsy team to not offer surgical resection to medically-intractable epilepsy patients. PNES: Psychogenic Non-Epileptic Seizures.

Multivariate logistic regression analysis was performed on all patients admitted to the EMU, incorporating the aforementioned variables identified on univariate analysis. Three independent predictors of surgical candidacy in patients admitted to the EMU were identified (Table 2). Male patients $(\mathrm{OR}=1.9,95 \% \mathrm{CI}=1.18$ 2.98, $\mathrm{p}=0.01$ ) and those with an identifiable lesion on MR imaging prior to EMU admission $(\mathrm{OR}=1.9,95 \% \mathrm{CI}=0.94-3.80$, $\mathrm{p}=0.07$ ) were more likely to be offered surgery. Additionally, inability to localize seizures based on routine scalp EEG prior to EMU admission was associated with a lesser likelihood of being offered resective surgery $(\mathrm{OR}=0.14, \mathrm{CI}=0.02-1.25, \mathrm{p}=0.08)$.

Based on the suggestion that the presence of a lesion on MRI and male gender were variables that could predict surgical candidacy, we sought to analyze this matter further; a summary of MRI findings in all patients (surgical and non-surgical) along with available pathological data from patients who underwent surgery has been provided in Table 3 . The majority of surgical patients were found to have either MTS $(105,41 \%)$ or normal MRI $(54,21 \%)$. Sixty one of the 105 surgical candidates $(58 \%)$ with MTS were male. Among the 155 non-surgical patients, the majority were found to have a normal MRI $(53,34 \%)$, findings suggestive of encephalomalacia/gliosis/atrophy (43, 28\%), or
MTS $(26,17 \%)$. Our database included 24 patients with a history of febrile seizures (11 female, 13 male). Sixteen (67\%) of these patients were found to have MTS on MRI, of which 13 were identified as possible surgical candidates. Pathology results were used to assess the accuracy of MRI findings. The majority of MTS cases were diagnosed correctly. Among 37 patients identified to have a "normal MRI" with available pathology, only $9(25 \%)$ were confirmed to have normal tissue while 18 $(50 \%)$ were found to have evidence of gliosis, $6(16 \%)$ had hippocampal sclerosis, 2 (5\%) had cortical dysplasia, and $2(5 \%)$ had evidence of neuronal heterotopias.

\section{Discussion}

In this single-institution, retrospective exploratory analysis of patients admitted to the EMU we identified three pre-admission variables that were associated with patients being identified as potential surgical candidates: Male gender, lesion on MR imaging, and localizing abnormality on pre-EMU outpatient EEG analysis. Together these variables can be used to guide the referral of patients and subsequent triage of admissions to the EMU.

The number of patients offered resective surgery in the current study $(62.5 \%)$ is considerably greater than previously reported. Benbadis and colleagues analyzed 253 patients admitted to the EMU for reasons including medication changes and clarification of epilepsy syndrome, and reported that only $23 \%$ were deemed surgical candidates ${ }^{9}$. Additionally, Villanueva and colleagues assessed 61 patients admitted to the EMU for surgical evaluation, of whom $36 \%$ were offered surgery. In their study, however, patients with temporal lobe epilepsy comprised only $56 \%$ of surgical cases ${ }^{10}$. Furthermore, the sample sizes in both studies were relatively small and the reasons for admission were variable; therefore, it is difficult to make any conclusive comparisons.

The leading reason for exclusion from surgical treatment was perceived futility of surgery. More than half of these patients were found to have seizures that were non-localizable/ lateralizable or multifocal/bilateral. This was followed by patients in whom the seizure foci were within or adjacent to eloquent cortex and patients who were adequately controlled on a trial of medications at the time of assessment. The remainder of patients had no electrographic seizures in the EMU or were diagnosed with PNES. While patients referred to the TWH EMU were identified as having medically-intractable epilepsy by their primary physician, it is possible that patient compliance or an inadequate trial of medications may have been an issue in those who were identified as responsive to medical therapy after assessment in the EMU. The rate of PNES among patients admitted to the EMU has been reported to range from 15.5$32.3 \%^{7,9}$ while an inability to detect EEG evidence of seizures was found to be $15 \%$ by Benbadis and colleagues, which they have acknowledged as unusually high secondary to non-specific referrals ${ }^{9}$. Therefore, the relatively lower rates of patients without electrographically-identifiable seizures and those with PNES in our EMU can be attributed to more specific referrals and stringent screening of referrals by our team of epileptologists.

Declining surgery, whether for iEEG implanation or resection of epileptogenic lesion, was found to comprise a significant 
Table 3: Summary of MRI and pathological findings in surgical and non-surgical candidates

\begin{tabular}{l|c|c|c}
\hline \multicolumn{1}{c|}{ MRI } & $\begin{array}{c}\text { Surgical candidates } \\
(\mathbf{n = 2 5 9 )}\end{array}$ & $\begin{array}{c}\text { Non-surgical candidates } \\
(\mathbf{n = 1 5 5})\end{array}$ & $\begin{array}{c}\text { Pathology results* } \\
(\mathbf{n}=\mathbf{2 0 2})\end{array}$ \\
\hline Normal & $54(21 \%)$ & $53(34 \%)$ & $10(5 \%)$ \\
\hline MTS $\quad 105(41 \%)$ & $26(17 \%)$ & $94(46 \%)$ \\
\hline$\quad$ History of febrile seizures** & 13 & 3 & \\
\hline$\quad$ Male & 61 & 10 & \\
\hline Encephalomalacia/ Gliosis/ Atrophy & $20(8 \%)$ & $43(28 \%)$ & 36 cases of gliosis $(18 \%)$ \\
\hline Neoplasm & $24(9 \%)$ & $12(8 \%)$ & $23(11 \%)$ \\
\hline Cavernoma & $27(10 \%)$ & $4(3 \%)$ & $12(6 \%)$ \\
\hline Other & $12(5 \%)$ & $10(6 \%)$ & $7(4 \%)$ \\
\hline
\end{tabular}

* Pathology results applicable only to patients undergoing surgery; ** A total of 24 patients within the database had a history of febrile seizures (13 were male); 16 were suggested to have MTS based on MRI results.

proportion of patients who did not undergo resective surgery. Twenty two percent of patients deemed as potential surgical candidates by the epilepsy team declined resective surgery which, along with those who refused surgery for iEEG implantation, constituted $40 \%$ of the 155 non-surgical patients. These data are comparable to proportions published by Carlson and colleagues ${ }^{11}$. Loss of patients to follow-up was also a leading factor among the non-surgical group of patients. Overall, 42 patients $(10 \%)$ lacked a complete follow-up, which is notably less than the 25 to $75 \%$ rate in the literature based on studies reporting outcomes ${ }^{11,12}$.

One unexpected finding of our study was the greater likelihood of males to go on to resective procedures. With regards to biological differences, idiopathic generalized epilepsy is known to be more common among females ${ }^{13}$; however, this was not specifically analyzed in our study. Furthermore, male gender has been identified as a predictor of a favorable outcome following surgery for patients with temporal lobe epilepsy without a definitive explanation for the difference in outcome ${ }^{14}$. While a biological explanation is a possibility, equity of access and underlying psychiatric state, neuropsychological profile, and other social issues influencing patient choice must also be considered. In our study, an equal number of males and females had been admitted to the EMU, while a slightly greater proportion of females $(58 \%)$ declined surgical treatment, which may have biased our results. The divide among patients with a history of febrile seizures or MTS on MRI was even as well, although a slightly higher number of males with MTS were identified as surgical candidates. It is, however, imperative that future studies examine the relationship between gender and an increased likelihood of undergoing resective surgery for epilepsy to validate and characterize the importance of this finding.

Patients with a lesion on MRI were more likely to be deemed surgical candidates. This is expected as the presence of a lesion on MRI has been linked to patients developing drug-resistant epilepsy; these patients are also most likely to benefit from surgery ${ }^{15-17}$. Conversely, poor surgical outcome is predicted in patients with epilepsy without a lesion on MRI ${ }^{18}$. While $79 \%$ of surgical candidates in our study had abnormalities demonstrated on MRI, the remainder of surgical candidates were identified as having normal MRI studies, which is in concordance with rates found in other studies ${ }^{19,20}$. On pathology, however, more than half of these cases were found to support an underlying pathological lesion such as gliosis. Therefore, from an institutional point of view, perhaps improvements on our 3-Tesla MR scanner or the detail with which images are read can be made.

Patients in whom the seizure source could not be localized on outpatient EEG analysis (based on inter-ictal EEG abnormalities) were seven times less likely to be surgical candidates. Intuitively, candidacy for resective epilepsy surgery requires a localizable epileptogenic zone; however this information may sometimes only be known after EMU admission.

Slightly more than half of patients with iEEG monitoring in our study were identified as surgical candidates. This is considerably lower than an earlier study by Zumsteg and Wieser, assessing candidates for selective amygdalohippocampectomy, in which $80 \%$ of patients were identified as surgical candidates following $\mathrm{iEEG}^{21}$. This difference is perhaps owing to improved non-invasive methods of assessment over time, a broader range of underlying pathology analyzed within our EMU, or inherent selection bias between different centers. At $15 \%$, the composition of total surgical candidates comprised of patients having undergone iEEG monitoring was similar to previously published data 22 .

On univariate analysis, a trend was identified with regards to a history of febrile seizures being identified as a potential predictor of resective surgical candidacy following iEEG monitoring (data not shown). While a prolonged history of febrile seizures is thought to result in hippocampal sclerosis in certain patients ${ }^{23}$, it is also possible that hippocampal abnormalities predispose patients to febrile seizures ${ }^{24,25}$. With regards to our study, $67 \%$ of patients with a history of febrile seizures were found to have MTS on MRI.

The findings of this exploratory study, once confirmed and validated, should inform patient screening for EMU admission, provide guidance for managing patient expectations during presurgical evaluations and serve as a starting point for the establishment of additional evidence-based guidelines. The 
presence of a lesion on MRI is strongly supportive of referral to an epilepsy center for a possible EMU admission. While the ability to localize a seizure focus on routine EEG analysis would be supportive of a referral, an inability to localize should not be a deterrent to the primary physician given that an assessment in the EMU is often necessary for this task. Although not all EMU referrals are for surgical assessment, this study re-emphasizes the need for a discussion with patients regarding potential surgical risks and outcomes while managing expectations and wishes, both prior to referral to the EMU and before proceeding with video-EEG monitoring.

This study was limited by its retrospective nature, preventing the possibility of having an independent validation group to determine the predictive ability of the variables. The pertinence of our study to a single tertiary center infers a referral bias; while it would be ideal to identify generalizable variables, each center has a different approach to identifying surgical candidates and broadly-applicable recommendations are not necessarily valid. While loss of patients to follow-up is common to most studies analyzing epilepsy patients, it is nonetheless a major limitation to our study as well. However, the percentage of patients lost to follow-up in our study was much lower than those in the literature, perhaps owing to the fact that our institution is one of only two major tertiary referral centers in our province with involvement of a multi-disciplinary epilepsy management team. Further limitations include using a sample of convenience and the likelihood that not all plausible variables (e.g. duration or frequency of seizures) were included in the database or analyzed. In addition, detailed analysis of EEG results was lacking. This study, however, represents an important exploratory analysis in a relatively large, consecutive cohort of patients. Further studies across multiple centers involving larger patient cohorts can elaborate on these data with the ultimate goal of optimizing the triage and admission of patients to the EMU.

\section{REFERENCES}

1. Schuele SU, Luders HO. Intractable epilepsy: management and therapeutic alternatives. Lancet Neurol. 2008;7(6):514-24.

2. Spencer S, Huh L. Outcomes of epilepsy surgery in adults and children. Lancet Neurol. 2008;7(6):525-37.

3. Engel J, Jr. Surgical treatments of the epilepsies. New York: Raven Press; 1993.

4. Spencer SS, Williamson PD, Bridgers SL, Mattson RH, Cicchetti DV, Spencer DD. Reliability and accuracy of localization by scalp ictal EEG. Neurology. 1985 Nov;35(11):1567-75.

5. Ibrahim GM, Barry BW, Fallah A, et al. Inequities in access to pediatric epilepsy surgery: a bioethical framework. Neurosurg Focus. 2012;32(3:E2).

6. Benbadis SR, Heriaud L, Tatum WO, Vale FL. Epilepsy surgery, delays and referral patterns-are all your epilepsy patients controlled? Seizure. 2003;12:167-70.

7. Smolowitz JL, Hopkins SC, Perrine T, Eck KE, Hirsch LJ, O'Neil M. Diagnostic utility of an epilepsy monitoring unit. Am J Med Qual. 2007 Mar-Apr;22(2):117-22.

8. Benbadis SR, Siegrist K, Tatum WO, Heriaud L, Anthony K. Shortterm outpatient EEG video with induction in the diagnosis of psychogenic seizures. Neurology. 2004 Nov 9;63(9):1728-30.

9. Benbadis SR, O'Neill E, Tatum WO, Heriaud L. Outcome of prolonged video-EEG monitoring at a typical referral epilepsy center. Epilepsia. 2004 Sep;45(9):1150-3.

10. Villanueva V, Gutierrez A, Garcia M, et al. Usefulness of VideoEEG monitoring in patients with drug-resistant epilepsy. Neurologia. 2011 January - February;26(1):6-12.

11. Carlson C, Dugan P, French J. Progression to epilepsy surgery following pre-surgical evaluation. American Epilepsy Society (Abstracts). 2011

12. Tsai JJ, Cheng TJ. Status of follow-up among patients with epilepsy in epilepsy clinic. Jpn J Psychiatry Neurol. 1992 Jun;46(2):405-8.

13. McHugh JC, Delanty N. Epidemiology and classification of epilepsy: gender comparisons. Int Rev Neurobiol. 2008;83: 11-26.

14. Burneo JG, Black L, Martin R, et al. Race/ethnicity, sex, and socioeconomic status as predictors of outcome after surgery for temporal lobe epilepsy. Arch Neurol. 2006 Aug;63(8):1106-10.

15. Lerner JT, Salamon N, Hauptman JS, et al. Assessment and surgical outcomes for mild type I and severe type II cortical dysplasia: a critical review and the UCLA experience. Epilepsia. 2009 Jun;50 (6):1310-35.

16. Cossu M, Lo Russo G, Francione S, et al. Epilepsy surgery in children: results and predictors of outcome on seizures. Epilepsia. 2008 Jan;49(1):65-72.

17. Jeha LE, Najm I, Bingaman W, Dinner D, Widdess-Walsh P, Luders $\mathrm{H}$. Surgical outcome and prognostic factors of frontal lobe epilepsy surgery. Brain. 2007 Feb;130(Pt 2):574-84.

18. Bernasconi A, Bernasconi N, Bernhardt BC, Schrader D. Advances in MRI for 'cryptogenic' epilepsies. Nat Rev Neurol. 2011 Feb;7 (2):99-108.

19. Scott CA, Fish DR, Smith SJ, et al. Presurgical evaluation of patients with epilepsy and normal MRI: role of scalp video-EEG telemetry. J Neurol Neurosurg Psychiatry. 1999 Jan;66(1):69-71.

20. Cascino GD, Jack CR, Jr., Parisi JE, et al. Magnetic resonance imaging-based volume studies in temporal lobe epilepsy: pathological correlations. AnnNeurol. $1991 \mathrm{Jul} ; 30(1): 31-6$.

21. Zumsteg D, Wieser HG. Presurgical evaluation: current role of invasive EEG. Epilepsia. 2000;41 Suppl 3:S55-60.

22. Vonck K, Boon P, Achten E, De Reuck J, Caemaert J. Long-term amygdalohippocampal stimulation for refractory temporal lobe epilepsy. Ann Neurol. 2002 Nov;52(5):556-65.

23. Scott RC, King MD, Gadian DG, Neville BG, Connelly A. Hippocampal abnormalities after prolonged febrile convulsion: a longitudinal MRI study. Brain. 2003 Nov;126(Pt 11):2551-7.

24. Sanon NT, Desgent S, Carmant L. Atypical febrile seizures, mesial temporal lobe epilepsy, and dual pathology. Epilepsy Res Treat. 2012;2012:9.

25. Scantlebury MH, Heida JG. Febrile seizures and temporal lobe epileptogenesis. Epilepsy Res. 2010 Mar;89(1):27-33. 